\title{
Biocatalytic paradigms for unconventional information processing
}

\author{
Marcos Pita*a, b ${ }^{2}$ Evgeny Katz \\ * Corresponding Author \\ a: Instituto de Catalisis y Petroleoquimica, CSIC. C/ Marie Curie, 2. 28049 Madrid, \\ Spain. e-mail: marcospita@icp.csic.es \\ b: Department of Chemistry and Biomolecular Science and NanoBio Laboratory \\ (NABLAB), Clarkson University, Potsdam, New York 13699, USA
}

\section{Keywords}

Biocomputing, Enzyme Biocomputing, Biocatalysis, Boolean Operation, Enzyme Logic Gate

\begin{abstract}
Here we present an analysis of a new unconventional computing paradigm based on chemical reactions catalyzed by enzymes. This distinct concept hinges on the specificity of biocatalysts and selects chemical changes as information bits. The present review unveils the development of enzyme-based computing - from single Enzyme Logic Gates performing Boolean operations to some examples of concatenated networks. Inputs are defined as chemicals or as the biocatalyst, while the system output is read via optical or electrochemical means. The coupling of enzyme logic gates to signal-responsive interfaces is also developed, thus providing an approach to integrate enzymatic biocomputing into existing technology.
\end{abstract}

\section{Introduction}

Nowadays, computers are one of the most powerful tools available for mankind's progress and are present in almost every aspect of modern life. As with such ubiquitous and constantly developing technologies, there is always room for exploring new, 
unconventional ways to improve all possible options and limitations existing in the prevailing know-how. This is the relationship between regular computing and chemical computing: the latter one has become an exciting research field where chemical reactions play a role in controlling computational parameters. [1-3] The concept for chemical computing consists of a liquid mixture of chemicals where some reactions take place; the presence or absence of different chemicals will determine the computing information. [45] Catalysis plays an important role as it allows for the control of the chemical reactions by lowering the activation energy, thus facilitating transformations that otherwise would not occur. This control of chemical transformations through catalytic reactions can allow for the tailoring of reaction rates along with selection of the specific chemicals produced. This resembles the control found in semiconductor electronic transmission or magnetic dipole inversion, which are the physical phenomena governing the basic processes of conventional computing. Although catalytic reactions are typically performed in a bulk solution, interfacial catalysis can also take place. This fact allows for the collection of information provided by the chemical reactions through a solid support, which acts simultaneously as catalyst and as an information transducer. The ideal limit for chemical computing is the utilization of an algorithm based on a single molecule to solve the problem, [6] thus permitting miniaturization while reaching high computation levels using different molecules working in parallel. [7] This research field, although nascent, still shows promising future potential. [8]

Biochemical computing, also known as biocomputing, can be described as chemical computing where biological entities play a significant role. Biocomputing is mainly based either on DNA recognition processes [9-12] or enzyme-catalyzed reactions. [13-16] The DNA hybridization reaction can be used to perform single parallel computational operations of nucleotide sequences which can be scaled up to perform operations of higher complexity. [17, 18] Enzymatic biocomputing, a relatively recent development compared to DNA computing, presents an alternative direction. DNA computing aims at solving complex computational tasks in a single step with multiple parallel reactions, while enzymatic biocomputing aims at the signal processing of different biomarkers. Reading the information contained in those biomarkers separately can be complicated, 
therefore the use of a single interface to funnel pre-processed information is a remarkable advantage. This opens a field of applications that makes the enzymatic biocomputing interesting to be studied and developed.

In this article we will present a list of existing biocomputing elements based on enzymatic reactions performing Boolean operations, some examples of enzyme logic gates networks, and finally, the connection of these enzyme logic operations with different devices.

\section{Logic on Enzymatic Reactions}

Enzymes are proteins that catalyze a chemical reaction while taking one or two chemical compounds called substrates and produce a set of different compounds called products. Mimicking a Boolean operation with enzyme-catalyzed reactions requires defining the inputs, the outputs and the machinery of the logic operation. When these elements are established, we obtain a Boolean operation that can be defined as an enzyme logic gate (ELG). The inputs of an ELG can be chemicals (substrates for the enzyme), in this case the enzymes together with other substances such as cofactors or buffers work as machinery, or vice versa - the enzymes themselves can act as inputs while their substrates are the machinery of the ELG. This flexibility is interesting for in vivo applications due to the triggering of enzyme cascade by specific biomarkers. An input signal is defined as two different states, the non-active one, $\mathbf{0}$, and the active one, $\mathbf{1}$. The straightforward definition for a $\mathbf{0}$ input is the absence of that chemical. The input $\mathbf{1}$ is defined as a concentration of a specific chemical sufficient to give a well-differentiated signal considered as $\mathbf{0}$ or $\mathbf{1}$. However, it is sufficient if the $\mathbf{0}$ and $\mathbf{1}$ signals are concentrations that provide a distinguishable gap in the final output, which is a consequence of the biocatalytic information processing. Furthermore, the chemical reactions that occur in the ELG should meet a crucial criterion: to produce (or consume) a chemical or a physical signal only when the combination of inputs results in a clearly distinguishable output defined as $\mathbf{1}$ or $\mathbf{0}$. 
Following these premises, a series of ELG mimicking single Boolean operators such as AND, OR, XOR, InhibA, NAND, NOR have been developed. [19-21] In this first example, an AND gate is presented as follows: the machinery consists of a solution titrated to $\mathrm{pH} 7$, containing the chemicals sodium sulfate, sucrose and urea (Figure 1A). The enzymes Glucose Oxidase (GOx) and Invertase (Inv) are inputs $\mathbf{1}$ and $\mathbf{2}$ respectively, and the output is the acidification of the solution due to the production of gluconic acid. If only GOx (Input $\mathbf{1} \mathbf{0}$ ) is added to the system, the sucrose will remain non-reacted. If only the Inv (Input $\mathbf{0}$ 1) is added to the system, the enzyme will produce glucose, thus maintaining the $\mathrm{pH}$. Only when both enzymes (Input 1 1) are added to the system, glucose is produced in the presence of Inv while GOx oxidizes glucose to gluconic acid which results in a $\mathrm{pH}$ decrease to almost 4 (Figure $1 \mathrm{C}$ ). Similarly, an OR gate is presented working as follows: in this case, the machinery consists of sodium sulfate, glucose, ethyl butyrate and urea. For the OR gate, Input $\mathbf{1}$ is GOx, input $\mathbf{2}$ is Esterase (Est) and the output is the acidification of the solution (Figure 1B). When GOx is added to the system (Input $1 \mathbf{0}$ ) the glucose is oxidized to gluconic acid and the $\mathrm{pH}$ is lowered to 4. When Est is added to the system (Input 0 1) the ethyl butyrate is hydrolyzed to butyric acid, which decreases the $\mathrm{pH}$ to a value close to 4 . The addition of both enzymes (Input 1 1) yields the production of two acids that decrease the $\mathrm{pH}$ to the same level (Figure 1D). Both logic gates can also include urea in their machinery, which provides a Reset mechanism: the addition of a Reset enzyme, Urease, can catalyze the production of ammonia, which raises the $\mathrm{pH}$ to slightly alkaline values, resulting in the output value of 0. These enzyme logic gates able to change the $\mathrm{pH}$ have shown high applicability, as they can be coupled with signal responsive materials sensitive to $\mathrm{pH}$, for example, polyelectrolyte-modified nanoparticles, [19] pickering emulsions, [22] membranes, [23] and Indium Tin Oxide electrodes modified with poly(vinyl)-piridine (PVP). [24] The coupling of ELGs with signal responsive materials sensitive to $\mathrm{pH}$ changes is one of the most promising directions for this research field, however an extensive analysis is out of the scope of the present report. 
Other ELGs using enzymes as inputs result in an output defined as the production of a compound with a determined optical absorbance. These ELGs will promote a change in the color when the output is $\mathbf{1}$, but maintain a low optical density if the output is $\mathbf{0}$. [20] The AND gate shows a machinery that consists of a solution containing phosphate buffer at $\mathrm{pH}$ 7, glucose, oxygen, and 2,2'-azino-bis(3-ethylbenzthiazoline-6-sulphonic acid) (ABTS). Input $\mathbf{1}$ of the AND gate is the enzyme GOx and input $\mathbf{2}$ is Microperoxidase-11 (MP-11), a peptide chain composed of 11 amino acids linked to a heme group with peroxidase activity. When the system is exposed to the input combination $\mathbf{1 0}$, the only reaction that occurs is the oxidation of glucose to give gluconic acid and hydrogen peroxide, yielding no change in the absorbance. The input combination $\mathbf{0} 1$ introduces the MP-11, which does not produce an output due to the lack of $\mathrm{H}_{2} \mathrm{O}_{2}$. Only in the case of input 11 the combination is successful: GOx produces the hydrogen peroxide that MP-11 reduces to water by oxidizing ABTS. Oxidized ABTS gives a strong absorption at $\lambda=415$ $\mathrm{nm}$, defined as output $=\mathbf{1}$. An example of a XOR gate is presented by using the enzymes Alcohol Dehydrogenase $(\mathrm{AlcDH})$ and $\mathrm{MP}-11$ as inputs. The XOR machinery is composed of phosphate buffer, $\mathrm{NADH}, \mathrm{NAD}^{+}$, ethanol and $\mathrm{H}_{2} \mathrm{O}_{2}$. The output of the XOR gate is defined as the change (either positive or negative) of the absorption at $\lambda=340 \mathrm{~nm}$, which results from a change in NADH concentration. The system shows an initial absorbance due to the existence of NADH in the solution, which remains constant in the presence of input combination $\mathbf{0} 0$. If input 10 enters in the system, AlcDH oxidizes the ethanol by reducing $\mathrm{NAD}^{+}$to $\mathrm{NADH}$. This reaction brings an increase in the absorbance at the selected wavelength, giving an output $\mathbf{1}$. If the input is $\mathbf{0} \mathbf{1}, \mathrm{MP}-11$ reacts with the $\mathrm{NADH}$ and the $\mathrm{H}_{2} \mathrm{O}_{2}$ existing in the solution, the decrease in absorbance gives an output 1. If the input combination is $\mathbf{1} \mathbf{1}$, the two enzymes perform opposing reactions: AlcDH produces NADH while MP-11 consumes it. The balance of these reactions, under appropriate concentrations, gives a combination where the absorbance remains unchanged, thus giving an output $=\mathbf{0}$. Other gates $(\mathrm{OR}$ and InhibA) are presented in the article, but the key point is the forward step demonstrated via the immobilization of enzymes on glass as opposed to a bulk reaction. Immobilization of the enzyme is a critical step because it allows for the reutilization of the biocatalyst, gives stability to the 
system, and permits coupling with other interfaces, such as electrodes or sensitive surfaces.

Other single gate operations that have been achieved in enzyme biocomputing are NAND and NOR gates. [25] These logic operations have a special appeal in electronics, as they are "universal" gates used which can yield any other logic operation when combined with itself in one or other configuration. Chemically, the concatenation of NAND gates is not so straight forward because the output is typically a chemical different from the inputs, therefore a treatment is required in order to ensure that the output of the first gate serves as an input for the second identical gate. The approach to develop the NAND and NOR gate is modular: in the first step, an AND and an OR gate that give a specific product, glucose, are developed. The AND gate module is composed of the enzyme maltose phosphorylase, which only produces glucose when two chemicals are added: maltose and phosphate. The OR gate module is composed of two enzymes, Invertase and Amyloglucosidase, which produce glucose from a disaccharide, sucrose and maltose respectively, thus producing glucose with either of the inputs. The second module is an Inverter that transforms the glucose output $\mathbf{1}$ into $\mathbf{0}$. The inverter consists of a solution of HEPES buffer containing ethanol, $\mathrm{NAD}^{+}$and the enzymes GOx, MP-11 and AlcDH. This system is designed to produce NADH upon the oxidation of ethanol catalyzed by AlcDH, with NADH being the final output of the whole system. When glucose is produced in a previous module (either AND or OR) it it serves as an input to the inverter and oxidized by GOx. This reaction triggers the enzymatic cascade that re-oxidizes all the NADH generated by $\mathrm{AlcDH}$, inverting the previous output $\mathbf{1}$ to $\mathbf{0}$. This work also demonstrates the first attempt at amplification and conversion in enzymatic biocomputing: this system is able to duplicate the concentration of NADH and the conversion of the output signal $\mathrm{NADH}$ to one of the initial inputs, maltose.

Regarding applications of ELGs, a couple of logic gates have been developed to differentiate injuries occurring under traumatic circumstances, for example, car accidents or battlefield action. [26] The system presented shows two ELGs working in parallel in the same solution aiming at differentiating traumatic brain injury (TBI) from hemorrhagic shock (HS). The first ELG consists of an AND gate whose machinery is Lactate Oxidase 
(LOx) and Horseradish Peroxidase (HRP) as enzymes in a phosphate buffer solution together with oxygen (in equilibrium with the atmosphere). The first input is lactate, which is a general biomarker that shows a ca. 6-fold increase under traumatic conditions. The second input is the hormone norepinephrine, a trauma biomarker with a $10^{3}$-fold concentration increase under traumatic conditions. When input combination 11 (12 mM lactate and $3.5 \mu \mathrm{M}$ norepinephrine) is added to the machinery, LOx oxidizes lactate to pyruvate while producing $\mathrm{H}_{2} \mathrm{O}_{2}$. The latter product is taken by HRP and used to oxidize norepinephrine to norepi-quinone in significant amounts. This can be easily detected using spectrophotometric methods due to norepi-quinone's absorption band at $\lambda=465$ $\mathrm{nm}$. The output can also be measured via electrochemical means by monitoring the reduction of norepi-quinone to norepinephrine at $-0.25 \mathrm{~V} v s \mathrm{Ag} / \mathrm{AgCl}$ reference electrode. When the lactate input corresponds to the $\mathbf{0}$ logic level $(2 \mathrm{mM})$, LOx will oxidize the lactate, however the production of $\mathrm{H}_{2} \mathrm{O}_{2}$ is not sufficient to give a significant signal in the next step. Likewise, an input $\mathbf{0}$ of norepinephrine $(2 \mathrm{nM})$ added into the system remain below the detection limit, even when sufficient amounts of $\mathrm{H}_{2} \mathrm{O}_{2}$ are produced in situ by lactate oxidase. This logic gate is presented as a quick and easy detection method for TBI because can correlate the two fastest biomarkers to appear in blood when happens the injure. However, differentiating TBI from HS is needed, so, an Identity gate working in parallel is added to the system. This Identity gate consists of the enzyme glucose dehydrogenase $(\mathrm{GDH})$ along with the cofactor $\mathrm{NAD}^{+}$. The single input existing in this gate is glucose, which exhibits a 5-fold increase when HS occurs (from $4 \mathrm{mM}$ to $30 \mathrm{mM}$ ), but remains at the physiological level of $4 \mathrm{mM}$ under a TBI condition. The output can be monitored through NADH production either optically at $\lambda=340 \mathrm{~nm}$ or electrochemically via oxidation at $+0.75 \mathrm{~V}$. The AND gate will determine the traumatic condition, while the identity gate will differentiate between TBI and HS.

One of the great advantages of enzymatic biocomputing is that the output signals are chemicals that were not present in the solution before the logic operation. This attribute allows the concatenation of several logic gates yielding more advanced systems. Several examples of concatenation have been recently developed, for example, the consolidation of four inputs (NADH, Acetaldehyde, Glucose and Oxygen) into a single output through 
three AND logic gates, a FANOUT operation and an OR gate (Figure 2). [27] The output (production of gluconic acid) was defined as $\mathrm{O}=[(\mathrm{A}$ AND B) AND C] OR (C AND D) and was able to change the $\mathrm{pH}$ only when the operator gave 1 as the final output which resulted in the opening and closing of a polymer brush attached to an ITO electrode. The process was able to switch ON and OFF the electrochemical circuit, which was measured by cyclic voltammetry using ferrocyanide as electrochemical probe. Only with the use of three enzymes, alcohol dehydrogenase, glucose dehydrogenase and glucose oxidase, it has been possible to create a logic network able to analyze 16 combinations of inputs.

Together with the development of logic operations performed by enzyme-catalyzed chemical reactions, other common devices in regular computing have been mimicked with enzymatic reactions. One method of information storage used in conventional computing is the flip-flop memory. These devices are responsible for storing information in the form of bits, which can have two possible configurations: 1 or 0 . The present configuration should be stable in time, changed only when addressed and commanded by a previous computation, and reversible upon activation. Even the simplest flip-flop system, a Set-Reset memory unit, is challenging to perform with enzymatic reactions. This is because when the systems include two correlated states they trend to form a chemical loop. This yields a balance of both markers under an equilibrium state instead of an increase of one specific chemical responsible for a final well-defined state. However, it has been possible to demonstrate this type of a chemically complicated system. [28] A core enzymatic set-reset memory unit has been demonstrated and linked to two different biochemical pathways responsible for providing the information to be stored. The core enzymatic system was composed of two enzymes: horseradish peroxidase (HRP) and diaphorase (Diaph). The oxidative reaction biocatalyzed by HRP was activated via the addition of $\mathrm{H}_{2} \mathrm{O}_{2}$, while adding $\mathrm{NADH}$ triggered the reductive reaction biocatalyzed by Diaph. The oxidative process resulted in the oxidized state of the redox mediator while the reductive process resulted in the reduced state of the redox mediator. The presence of the reduced or oxidized mediator in the system could be detected by optical or electrochemical means, thus reporting on the state of the system. The oxidized state of the mediator was defined as digital $\mathbf{1}$, while the reduced state as 
digital $\mathbf{0}$. When the digital states are defined, the processes and signals resulting in these states become certain: The $\mathrm{H}_{2} \mathrm{O}_{2}$ input producing the oxidized state (1) is defined as set (S) and the NADH input yielding the reduced state $(\mathbf{0})$ is defined as reset $(\mathbf{R})$. Using this combination of enzymes it was possible to store the information given by other biochemical paths that yield the final production of the Set-Reset chemical inputs. The core system was extended one additional step in which glucose oxidase (GOx) could generate $\mathrm{H}_{2} \mathrm{O}_{2}$ through the oxidation of the glucose, which acts as the "set" input. The reset path was given by alcohol dehydrogenase $(\mathrm{AlcDH})$, responsible for the oxidation of ethanol while reducing $\mathrm{NAD}^{+}$to $\mathrm{NADH}$, which resulted in ethanol acting as the "reset" input. The mediator responsible for the optical output storage signal was dichloroindophenol (DCIP). When the "set" signal (glucose) is applied, the resultant oxidation of DCIP, shows an increase in the absorption band centered at $\lambda=600 \mathrm{~nm}$. Application of the "reset" signal (ethanol) yields the reduction of DCIP along with the appearance of $\mathrm{NADH}$, which shows an absorption peak at $\lambda=340 \mathrm{~nm}$. The concentrations of chemicals were properly balanced to keep the system reversible. Another example of the flip-flop performance was achieved when the reset signal was given by glucose-6phosphate dehydrogenase (G6PDH) using D-glucose-6-phosphate as "reset" primary input. In this example, the $\mathrm{H}_{2} \mathrm{O}_{2}$ signal was generated by lactate oxidase (LOx) in the presence of lactate as the primary "set" input. The optical responses of the system were similar to those in the previous example. The Set-Reset flip-flop core formed by HRP and Diaph was also measured electrochemically by the use of an electrochemically active mediator, ferrocyanide. [28]

\section{Enzyme Logic Gates interacting with materials}

The successful demonstration of enzyme-catalyzed systems with built-in logic can appear trivial, however it provides a vital component for the construction of bio-computing hierarchical structures. These logic systems should interact with materials and connect through interfaces with actuators, switches, electrodes, etc. in order to perform higher functions as opposed to being “just a chemical solution". One interesting approach is the 
immobilization of redox enzymes on the surface of an electrode which allows for the transport of electrons to or from an electronic interface. In this environment, the electrodes can perform two different functions: serve an electronic input to the system and provide a simultaneous read-out. The first example of enzyme logic gates wired to an electrode was published in the recent years. [29] The system was built by first immobilizing a rather complex molecule to a gold electrode via self-assembly. This molecule is comprised of a thiolated amino acid, cistamine, bound to a pyrroloquinoline quinone (PQQ) radical, which is also covalently attached to a flavin-adenine dinucleotide (FAD) molecule. These three components will have different functions at the biointerface: the cistamine will perform the self-assembly to the gold electrode through its thiol group, while the FAD is the cofactor of the enzyme GOx. Once the selfassembled monolayer is formed on the electrode it can be used to attach by affinity a GOx apoenzyme, thus reconstituting the enzyme on the surface of the electrode while retaining redox activity. The PQQ acts as a linker and also serves the function of electron transferring, or "wiring" the enzyme to the electrode. Once the GOx is attached to the gold electrode surface, it offers a highly functionalized surface to the solution. The enzyme contains several lysine amino acids, thus providing primary amino groups used as convalent linkers for a second biocatalytic entity. Microperoxidase (MP-11) is a pseudo-enzyme composed of a hemin group attached to a short 11-peptide chain, which includes several carboxylic groups - these carboxylic groups are easily bound to the lysine radical using a carbodiimide coupling protocol. In the end, what is achieved is an electrode with two biocatalysts immobilized on the surface, which can perform different redox reactions. Both enzymes are connected to the electrode surface, while retaining biocatalytic independence. The biofunctionalized electrode can perform three electrochemical reactions: The oxidation of glucose by GOx, the oxidation of $\mathrm{H}_{2} \mathrm{O}_{2}$ by MP-11, and the reduction of $\mathrm{H}_{2} \mathrm{O}_{2}$ by MP-11. The key component of the system is that the oxidation of glucose occurs at a potential lower than the reduction of hydrogen peroxide, so it is possible to reach a point where the electrochemical processes can cancel each other. To perform logic the operations the biofunctionalized electrode is considered as the machinery, and the chemicals glucose and $\mathrm{H}_{2} \mathrm{O}_{2}$ are the inputs $\mathbf{1}$ and $\mathbf{2}$, respectively. The output of the system is defined as a generated current threshold with 
different three read-out potentials and, depending on the selected potential, a different logic operation will result. The most reductive potential $(-0.1 \mathrm{~V}$ vs $\mathrm{Ag} / \mathrm{AgCl}$ ref. electrode) used is negative enough to prevent the oxidation of glucose by GOx. This yielded an Inhibit A-type logic gate; input 1 (glucose) is oxidized by GOx, however the negative potential applied to the electrode prevents the transfer of electrons from the enzyme's redox center. On the other hand, if input 2 reaches the system, the potential induces the reduction of hydrogen peroxide catalyzed by MP-11, thus generating a decrease in the detected current. If both inputs are applied, the system behaves exactly as if only $\mathrm{H}_{2} \mathrm{O}_{2}$ were applied, configuring the Inhibit-A system. If the read-out potential is very positive $(+0.45 \mathrm{~V})$, the system will offer an OR behavior. At this potential glucose is easily oxidized by GOx, and MP-11 also can oxidize $\mathrm{H}_{2} \mathrm{O}_{2}$, so whenever any or both of the inputs reach the system a positive current increase will occur. The most challenging read-out is when the applied potential is $+0.18 \mathrm{~V}$ - between the two previous potentials. In this case, the system behaves as a XOR gate: when glucose is added there is an increase in the oxidative current, when the $\mathrm{H}_{2} \mathrm{O}_{2}$ is added there is an increase in the reductive current, and when both inputs simultaneously reach the system they balance each other producing a negligible current. Therefore, there is only a current increase for the 10 and on $\mathbf{0} 1$ input combinations. The example shows a flexible configuration where several enzyme logic gates can be achieved with the same system, and could serve as a platform to design analogs performing other logic operations. The challenging task is to develop strategies to achieve direct electron transfer from the enzymes to the electrode surface.

The interaction of enzyme logic gates with electro-active surfaces has been one of the most successful approaches. Electrode surfaces can be modified not only to anchor the enzymes, but also to add a responsive material such as a polyelectrolyte. This material provides an interface capable of changing conformation, and consequently its properties, depending on the environment. For example, the polymer poly(vinyl)pyridine (PVP) is sensitive to $\mathrm{pH}$. [30] When the $\mathrm{pH}$ is acidic enough (below 4.5) the polymer chains become protonated and hydrophilic, acquiring a swollen configuration due to a local increase in osmotic pressure. In this case, the electrode would be open for negatively 
charged redox species. On the other hand, if the $\mathrm{pH}$ is not acidic enough (above 5.5), the polymer deprotonates, becomes hydrophobic, and collapses on the surface, preventing the diffusion of most chemical probes (although the hydrophobic environment is still permeable to some organic probes like thionin). Another advantage of the PVP brush is that it can act as a scaffolding for redox active mediators, for example Osmium pyridinetype aromatic complexes in the polymer chain. These complexes will only be electrochemically active when the polymer is swollen, because under shrunken configuration prevents the diffusion of Os complexes to the surface. This Os complex has been able to catalyze the oxidation of glucose by GOx in the absence of oxygen, but only when the polymer is swollen. [30]

As previously described, it is possible to control the $\mathrm{pH}$ of the solution using enzymatic reactions that produce acids or bases. Coupling these enzymatic reactions to electrodes allows for the switching ON and OFF of electrochemical processes. This approach utilized two parallel biochemical reactions to control the performance of an Os-PVP modified electrode. [31] The electrode is connected to GOx by accepting the electrons produced in the oxidation of glucose under anaerobic conditions; this is possible because the Os complex present in the modified electrode acts as redox mediator. The second biochemical system is responsible for the $\mathrm{pH}$ control. Acidification occurs by the production of butyric acid from ethyl butyrate catalyzed by the enzyme Esterase (Est?). This reaction has its antagonist in the production of ammonia from urea catalyzed by Urease. These two processes are an enable-reset system, able to open and close the modified electrode by acidifying or neutralizing the solution. Only when the electrode is open the oxidation of glucose by GOx is allowed.

A different configuration is used to facilitate the $\mathrm{pH}$ changes through ELGs. [32] In this case, the final output product should change the proton concentration in the solution. The $\mathrm{pH}$ change is detected with a PVP-modified electrode. The AND gate designed for this purpose utilized Inv and GOx as machinery, in a non-buffered environment. The inputs for this AND gate are sucrose and oxygen, and the output is gluconic acid. Given that the solution is non-buffered, the gluconic acid can easily lower the $\mathrm{pH}$ below 4.5, the point 
where the polymer changes its conformation. Only when oxygen and sucrose are added to the system (Input 1 1), gluconic acid is produced. If there is only sucrose (Input 1 0), Inv will produce glucose, however GOx does not have an electron acceptor available to complete the redox process. Likewise, if there is only oxygen present (Input $\mathbf{0}$ 1), electrons are not available to be transferred. An OR gate coupled to this electrode was designed using GOx and Esterase to lower the $\mathrm{pH}$. In this case, the inputs are glucose and ethyl butyrate, with oxygen being part of the machinery. When glucose is added to the system, GOx produces gluconic acid yielding a $\mathrm{pH}$ decrease. The same solution acidification results if ethyl butyrate is added to the system; Est produces butyric acid and the $\mathrm{pH}$ lowers. If both inputs enter the system, again, there is an acidification of the media. For both logic operations, when the output is $\mathbf{1}$ ( $\mathrm{pH}$ acidification) the electrode opens and allows electrochemical processes such as the oxidation of NADH, a biochemical species with redox activity. The process can be detected using different electrochemical techniques, for example cyclic voltammetry or impedance spectroscopy. Finally, reversibility of the polymeric switch is demonstrated by using the reset function mentioned above: Urease is present in the machinery of the logic gates, and a reset input of urea yields the production of ammonia; alkalinization of the $\mathrm{pH}$ causes the PVP brush to collapse to the surface of the electrode, thus impeding the electron transfer process. Once the pH-dependent electron transfer process is developed, the electrochemical readout is possible to expand the enzyme logic gate network, connecting several operations and analyzing a relatively larger amount of biochemical inputs (Figure 2). [27] The switchable electrode and its electrochemical processes are not only a method of detection and transduction, this approach can also be applied as either the anode or cathode of a biofuel cell, however, the switches to control biofuel cells are a rather extensive area and are out of the scope of the present review. [33]

These AND, OR and Reset gates described above and designed to control the $\mathrm{pH}$ were also coupled to Field-effect silicon chips, which could provide an electrical output signal in terms of capacitance variation. [34] A Silicon chip prepared on an aluminum layer is functionalized with thiol groups via a reaction with a thiolated silane coupling agent. The presence of the thiol groups allows the self-assembly of gold nanoparticles on the 
semiconductor surface. Once the nanoparticles are placed on the surface, 3mercaptopropionic acid is added to the system, forming a self-assembled monolayer $(\mathrm{SAM})$ which can protonate and deprotonate upon changes in the external $\mathrm{pH}$. The action of the logic gates yields either $\mathrm{pH}$ acidification (enzyme logic gate output $=\mathbf{1}$ ) or the $\mathrm{pH}$ remains unchanged (enzyme logic gate output $=\mathbf{0}$ ). Given that the silicon chip is a semiconductor, a voltammetric analysis will not yield a significant result, however impedance spectroscopy is a very sensitive tool. It was shown that the $\mathrm{pH}$ changes given by any of the AND/OR logic gates could be detected as a shift in capacitance of approximately $1 \mathrm{nF} \cdot \mathrm{cm}^{-2}$. The application of the Reset signal returned the system to its initial capacitance, demonstrating the system's reversibility.

As for any biotechnological research area, the immobilization of enzymes will bring several advantages such as stability and reusability. Several approaches for ELGs comprised of immobilized enzymes have been already tested. The AND and OR ELGs used for controlling the PVP-modified electrode [30] have been immobilized on silica microparticles via glutaric dialdehyde coupling. [35] In this case the read-out of the output was detected via optical means. Magnetic coregold-shell nanoparticles modified with a self-assembled monolayer of 3-mercaptopropionic acid (MPA) were present in the system. These nanoparticles show a localized surface plasmon resonance (LSPR) band centered at $\lambda=535 \mathrm{~nm}$. The decrease of the $\mathrm{pH}$ to 4 reaches the $\mathrm{pKa}$ of the immobilized MPA, and the protonation of the carboxylate groups partially depletes the electron density on the nanoparticles' surface. The resulting effect is the shift of the LSPR to a less-energetic wavelength, $\lambda=560 \mathrm{~nm}$. The system also presents immobilized urease, therefore the built-in reset function is activated with the addition of urea. In this case, the carboxylic groups deprotonated and decreased the LSPR wavelength. Dynamic light scattering analysis showed that the shift in the LSPR was due to the protonation/deprotonation of the carboxylic acid group and not to the aggregation of the nanoparticles.

Immobilization of the enzymes on conductive nanoparticles can yield an electrochemical transducer, thus granting a higher degree of applicability compared to an optical transducer. $25 \mathrm{~nm}$ magnetic core-gold shell nanoparticles have been used as a conductive 
scaffold to immobilize enzymes. [36] The discussed work presents the enzyme GOx immobilized on the magnetic-gold nanoparticles via EDC coupling. Next, the enzymemodified nanoparticles are magnetically collected on the surface of a PVP-modified Indium Tin oxide (ITO) electrode. The solution used in this system was a weak buffer that maintained the bulk $\mathrm{pH}$ close to neutral values. The engineered system changed the $\mathrm{pH}$ of the solution only in the local surrounding of the electrode when glucose was provided, which was oxidized by GOx to gluconic acid. The PVP was protonated without a significant $\mathrm{pH}$ change in the bulk solution, achieving increased electrochemical activity. The GOx-modified nanoparticle electrode presents a step toward operating as an electrochemical transducer for enzyme logic gates under physiological environment, which is the most promising direction for unconventional computing systems.

\section{Conclusions}

A new unconventional computing paradigm based upon chemical reactions catalyzed by enzymes is revealed and discussed in the present article. Several logic operations including AND, OR, XOR, NAND, and NOR are defined and shown to properly perform computing operations. The existing systems utilize model enzymes for the proof of concept as well as applicable systems for processing biological information during physiologically traumatic situations. Information storage by means of biocatalyzed

chemical reactions is also revealed as possible. Optical and electrochemical interfaces have been tuned to be connected with the ELGs' output, introducing a step forward toward the control of biointerfaces with the enzyme logic operations. All these facts yield the emergence of an interesting research field with promising practical applications, still in a rather early stage of growth and development.

\section{Acknowledgements}

Authors would like to thank Guinevere Strack for critical discussion of the present work. M. Pita wishes to thank the funding support provided by Spanish MICINN Ramon y 
Journal of Computational and Theoretical Nanoscience 8: 401- 408 (2011)

Cajal 2009 Research Funding Programme. This work was supported by the National Science Foundation (grants CCF-0726698, DMR-0706209) and by the Office of Naval Research (ONR Award \#N00014-08-1-1202).

\section{References}

1. C. Teuscher and A. Adamatzky (eds.), Unconventional Computing 2005: From Cellular Automata to Wetware, Luniver Press (2005).

2. A. Adamatzky and C. Teuscher (eds.), From Utopian to Genuine Unconventional Computers, Luniver Press (2006).

3. Y. M. Yin and X. Q. Lin, Progress in Chemistry 13, 337 (2001).

4. A. Adamatzky, B. De Lacy Costello, and T. Asai, Reaction-Diffusion Computers, Elsevier Science (2005).

5. A. Adamatzky, Computing in Nonlinear Media and Automata Collectives, Taylor \& Francis (2001).

6. T. Sienko, A. Adamatzky, N. G. Rambidi, and M. Conrad (eds.), Molecular Computing, MIT Press (2005).

7. A. Adamatzky, IEICE Trans. Electronics E87C, 1748 (2004).

8. G. Bell and J. N. Gray, Beyond Calculation: The Next Fifty Years of Computing, edited by P. J. Denning and R. M. Metcalfe, Copernicus/ Springer (1997), Chap. 1, p. 30.

9. L. M. Adleman, Science 266, 1021 (1994).

10. G. Paun, G. Rozenberg, and A. Salomaa, DNA Computing-New Computing Paradigms, Springer (1998).

11. Z. Ezziane, Nanotechnology 17, R27 (2006).

12. N. Jonoska, J. Computer Sci. Techn. 19, 98 (2004).

13. R. Baron, O. Lioubashevski, E. Katz, T. Niazov, and I. Willner, Angew. Chem. 45, 1572 (2006).

14. R. Baron, O. Lioubashevski, E. Katz, T. Niazov, and I. Willner, Org. Biomol. Chem. 4, 989 (2006).

15. R. Baron, O. Lioubashevski, E. Katz, T. Niazov, and I. Willner, J. Phys. Chem. A 110, 8451 (2006).

16. T. Niazov, R. Baron, E. Katz, O. Lioubashevski, and I. Willner, Proc. Natl. Acad. USA 103, 17160 (2006).

17. L. Kari, G. Gloor, and S. Yu, Theoretical Computer Science 231,192 (2000).

18. J. Watada, S. Kojima, S. Ueda, and O. Ono, Int. J. Innov. Comp. Inf. 2, 273 (2006).

19. M. Motornov, J. Zhou, M. Pita, V. Gopishetty, I. Tokarev, E. Katz, and S. Minko. Nano Lett. 8 (9), 2993 (2008).

20. G. Strack, M. Pita, M. Ornatska, and E. Katz, ChemBioChem 9, 1260 (2008).

21. J. Zhou, M. A. Arugula, J. Halámek, M. Pita, and E. Katz. J. Phys. Chem. B 113, 16065 (2009).

22. M. Motornov, J. Zhou, M. Pita, I. Tokarev, V. Gopishetty, E. Katz, and S. Minko. Small 5 (7), 817 (2009). 
23. I. Tokarev, V. Gopishetty, J. Zhou, M. Pita, M. Motornov, E. Katz, and S. Minko. ACS Appl. Mater. Interfaces 1 (3), 532 (2009).

24. J. Zhou, T. K. Tam, M. Pita, M. Ornatska, S. Minko, and E. Katz. ACS Appl. Mater. Interfaces 1 (1), 144 (2009).

25. J. Zhou, M. A. Arugula, J. Halámek, M. Pita, and E. Katz. J. Phys. Chem. B 113, 16065 (2009).

26. K. M. Manesh, J. Halámek, M. Pita, J. Zhou, T. K. Tam, P. Santhosh, M. Chuang, J. R. Windmiller, D. Abidin, E. Katz, J. Wang. Biosens. Bioelectr. 24, 3569 (2009).

27. M. Privman, T. K. Tam, M. Pita, and E. Katz. J. Am. Chem. Soc. 131, 1314 (2009).

28. M. Pita, G. Strack, K. MacVittie, J. Zhou, and E. Katz. J. Phys. Chem. B 113, 16071 (2009).

29. M. Pita and E. Katz. J. Am. Chem. Soc. 130, 36 (2008).

30. T. K. Tam, M. Ornatska, M. Pita, S. Minko, and E. Katz. J. Phys. Chem. C 112, 8438 (2008).

31. T. K. Tam, J. Zhou, M. Pita, M. Ornatska, S. Minko, and E. Katz. J. Am. Chem. Soc. 130, 10888 (2008).

32. J. Zhou, T. K. Tam, M. Pita, M. Ornatska, S. Minko, and E. Katz. ACS Appl. Mater. Interfaces 1, 144 (2008).

33. E. Katz, and M. Pita. Chem. Eur. J. 15, 12554 (2009).

34. M. Krämer, M. Pita, J. Zhou, M. Ornatska, A. Poghossian, M. J. Schöning, and E. Katz. J. Phys. Chem. C 113, 2573 (2009).

35. M. Pita, M. Krämer, J. Zhou, A. Poghossian, M. J. Schöning, V. M. Fernandez, and E. Katz. ACS Nano 2, 2160 (2008).

40. M. Pita, T. K. Tam, S. Minko, and E. Katz. ACS Appl. Mater. Interfaces 1, 1166 (2009).

\section{Figure Captions}

Figure 1. Enzyme Logic Gates operating with enzymes as inputs. (A) Operation of an AND logic gate with glucose oxidase and invertase as inputs. (B) Typical normalized output of the AND gate. (C) Operation of an OR logic gate with glucose oxidase and esterase as inputs. (D) Typical normalized output of the OR gate. The grey bars mark the thresholds to limit 0 output and 1 output.

Figure 2. Enzyme logic network including three different enzyme logic gates and four inputs coupled to a $\mathrm{pH}$-dependent signal response electrode. 


\section{Biographies}

Marcos Pita obtained Ph.D. in 2006 after his research in Catalysis Institute (Madrid) belonging to the Spanish Research Council. During his Ph.D. study Pita was also a visiting researcher in Van't Hoff Laboratorium at Utrecht University and Analytical Chemistry Department at Lund University. From 2007 to 2009 he joined Clarkson University as a Research Assistant Professor researcher in the laboratory of Prof. Katz. In 2010 he joined the Catálisis Institute as a Ramon y Cajal Researcher. His research interests are concentrated in the development of nanostructured materials for biosensor and bioelectronic applications. He is deeply involved in the development of various biocomputing systems integrated with signal responsive materials. He has published more than 55 articles in peer-reviewed journals and holds a world patent on the synthesis of nanoparticles for biosensor applications.

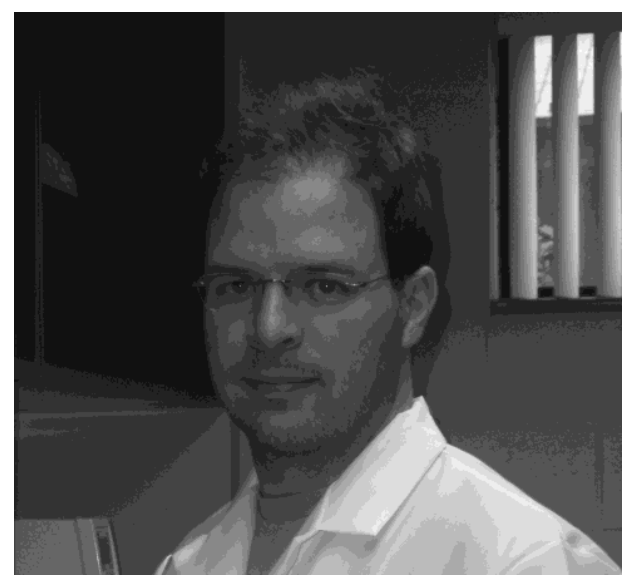

Evgeny Katz received Ph.D. from Frumkin Institute of Electrochemistry (Moscow), Russian Academy of Sciences, in 1983. He was a senior researcher in the Institute of Photosynthesis (Pushchino), Russian Academy of Sciences, in 1983-1991. In 1992-1993 he performed research at München Technische Universität (Germany) as a Humboldt fellow. Later, in 1993-2006, Dr. Katz was a research associate professor at the Hebrew University of Jerusalem. From 2006 he is Milton Kerker Chaired Professor at the Department of Chemistry and Biomolecular Science, Clarkson University, NY. He published more than 290 papers in peer-reviewed journals with the total citation more than 15,000 and holds 20 international patents. He serves as a Editor-in-Chief for IEEE Sensors Journal. His scientific interests are in the areas of bioelectronics, biosensors and biofuel cells. Currently he is actively involved in the research in biocomputing, signalresponsive materials and their applications in logically operating biosensors. 
Journal of Computational and Theoretical Nanoscience 8: 401- 408 (2011)

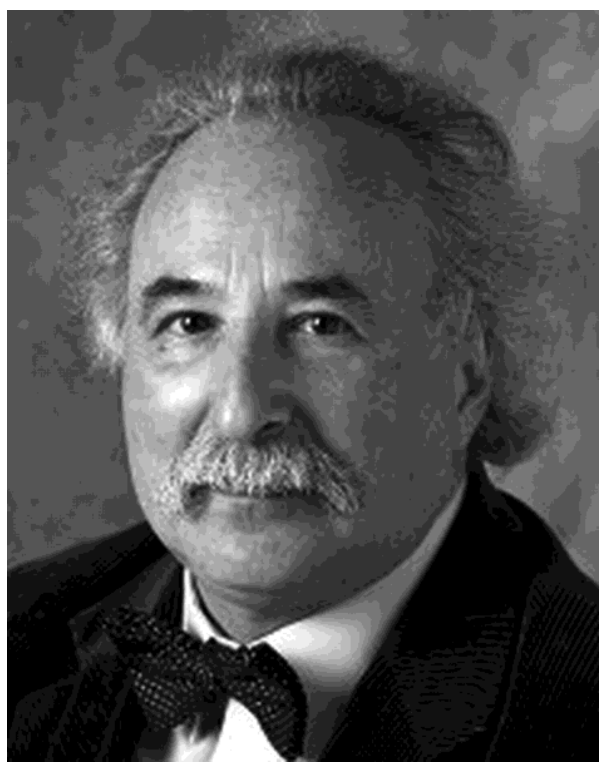




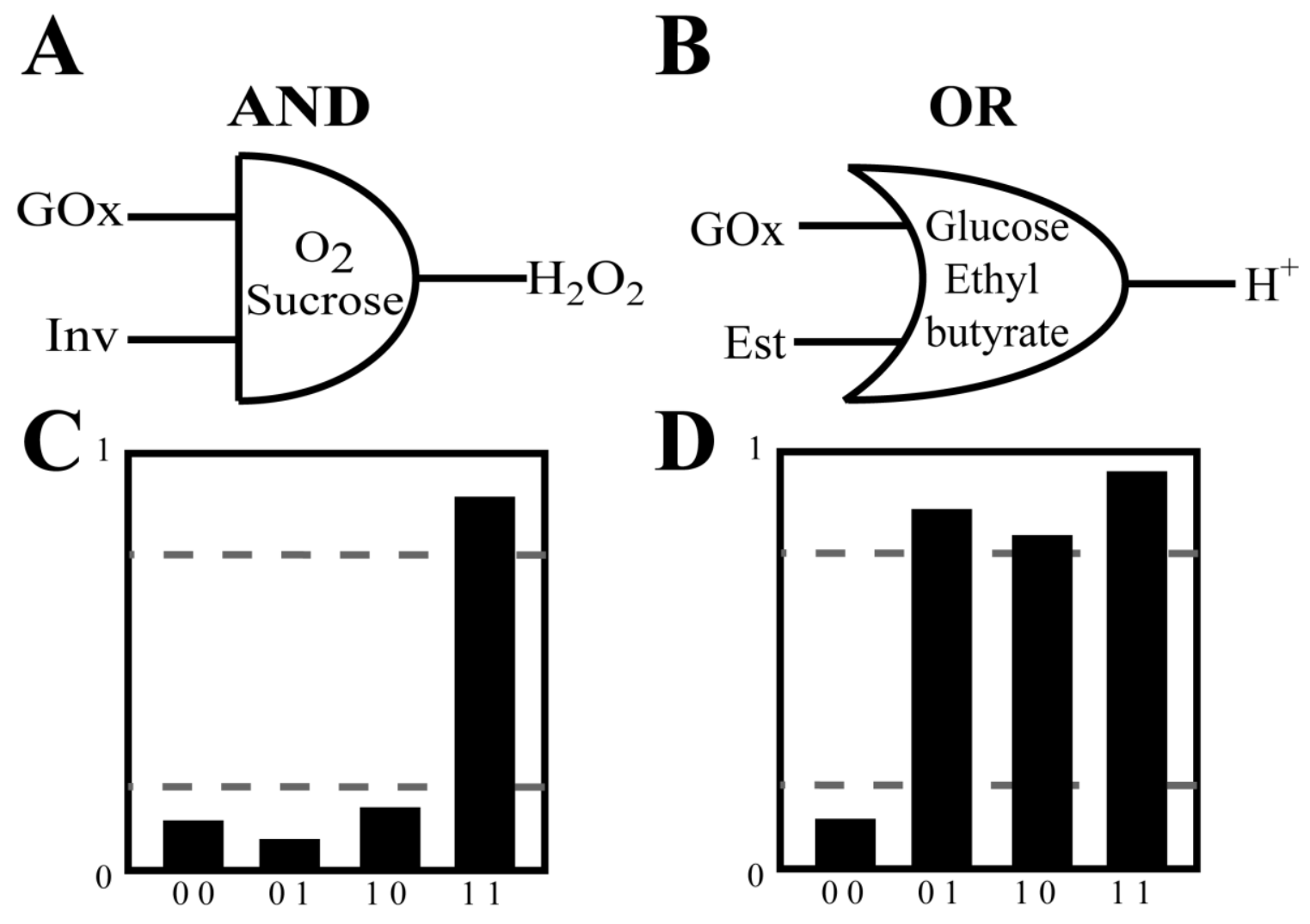

Figure 1. 
Journal of Computational and Theoretical Nanoscience 8: 401 - 408 (2011)

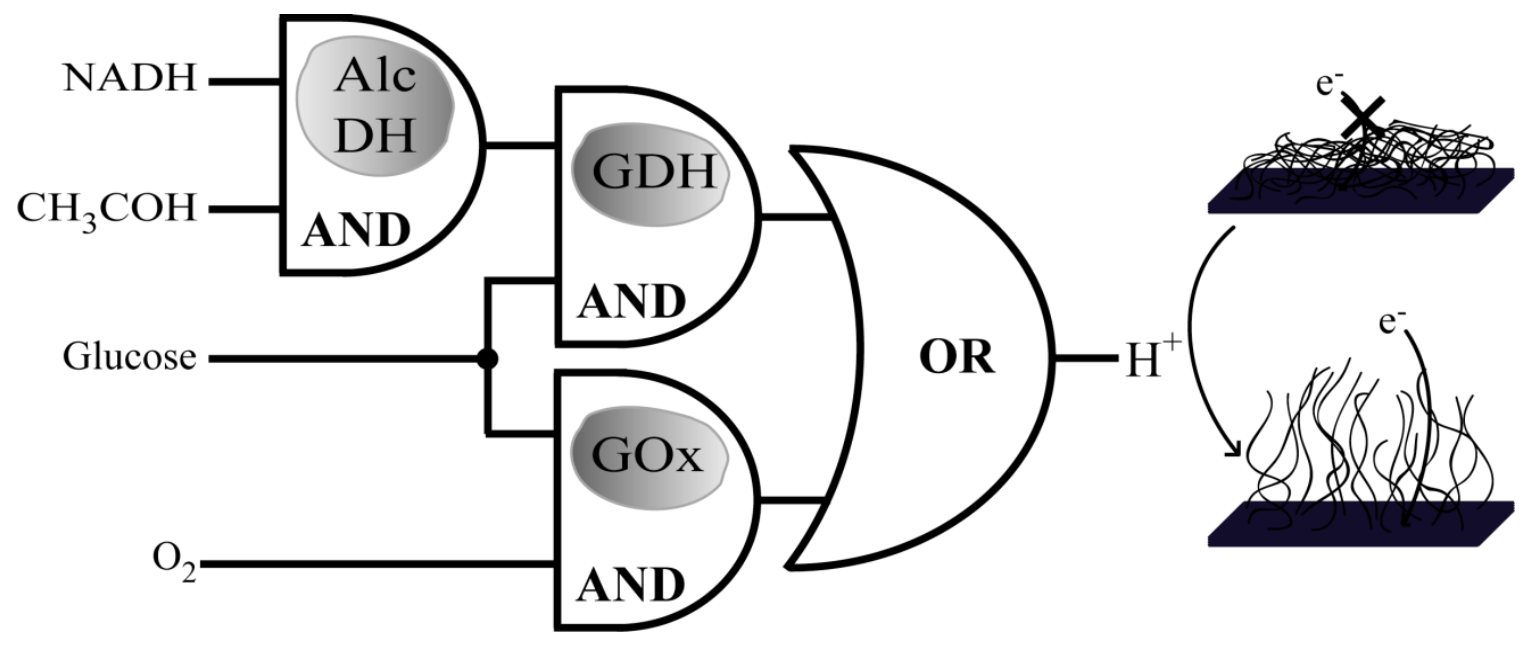

Figure 2. 